\title{
Beyond the Standard Model physics prospects at the Deep Underground Neutrino Experiment
}

\section{Doojin Kim*a,*}

${ }^{a}$ Mitchell Institute for Fundamental Physics and Astronomy, Department of Physics and Astronomy, Texas A\&M University

College Station, TX 77843, USA

E-mail: doojin.kim@tamu.edu

The Deep Underground Neutrino Experiment (DUNE) is an international project for neutrino physics and proton-decay searches, currently in the design and planning stages. Once built, DUNE will consist of two detectors exposed to the world's most intense neutrino beam. The near detector at Fermilab will record neutrino interactions near the beginning of the beam line. The other, much larger, detector, comprising four 10-kton liquid argon time projection chambers (LArTPCs), will be installed at a depth of $1.5 \mathrm{~km}$ at the Sanford Underground Research Facility in South Dakota, about 1,300 km away from the neutrino source. The unique combination of the high-intensity neutrino beam, DUNE's high-resolution near detector system, and massive LArTPC far detector enables a variety of probes of Beyond-the-Standard-Model (BSM) physics, from the discovery of new particles (sterile neutrinos or dark matter), to precision tests of beyond the three-flavor mixing paradigm, non-standard neutrino interactions (NSIs), heavy neutral leptons, and the detailed study of rare processes (e.g., neutrino trident production). This article reviews these physics topics and discusses the prospects for their discovery at the DUNE experiment.

40th International Conference on High Energy physics - ICHEP2020

July 28 - August 6, 2020

Prague, Czech Republic (virtual meeting)

\footnotetext{
*for the DUNE Collaboration

*Speaker
} 


\section{Introduction}

The DUNE is a next-generation neutrino experiment. DUNE will be sitting at the forefront of neutrino-sector physics and is expected to uncover various interesting phenomena. The DUNE science program includes long-baseline neutrino oscillations (e.g., leptonic CP violation, neutrino mass ordering, and precision tests of the 3-neutrino mixing framework) and detection of supernova neutrinos. DUNE's capability is not restricted to neutrino-sector physics, but allows us to study rare proton decays and perform searches for BSM physics. DUNE utilizes a high-intensity, high-energy proton beam (beam energies of $60-120 \mathrm{GeV}$ and $\sim 10^{21}$ protons on target per year), creating not only high-intensity neutrino fluxes but new physics signals. Furthermore, large-volume detectors can be sensitive to neutrino and new physics signals coming from astrophysical sources including the sun and galactic/extra-galactic sources.

Once built, DUNE will have detector complexes both at the far site and the near site which will allow for various physics opportunities. The far detector with four 10-kton (fiducial mass) modules employs LArTPC technology, featuring a low energy threshold and high resolution in energy, angle, and position, allowing unrivaled particle detection and identification. When it comes to the near detector, there are three sub-detectors: a liquid argon TPC, a Multi-Purpose Detector, and a System for on-Axis Neutrino Detection. The first two can be moved sideways 33 meters (DUNE-PRISM) [1], making them particularly useful for new physics searches.

In this article, we briefly overview physics prospects at DUNE, particularly focusing on BSM physics cases. The DUNE BSM effort covers many interesting topics. Examples include nonstandard short-baseline and long-baseline neutrino oscillations (e.g., sterile neutrino mixing, NSIs of neutrinos, and CPT violation), search for beam-induced new phenomena at the near detector (e.g., neutrino tridents and low-mass dark matter), and new cosmic-origin phenomena at the far detector (e.g., boosted dark matter). More extensive discussions of the BSM topics including other physics cases are also available in a Technical Design Report [2] and a DUNE BSM physics paper [3].

\section{BSM in Non-Standard Neutrino Oscillations}

In this section, we discuss new physics discoverable in neutrino oscillations, beginning with the sterile neutrino. Sterile neutrinos are predicted in many BSM models to explain the origin of neutrino masses, and the discovery of sterile neutrinos may provide insight into see-saw mechanisms. Basically, the potential mixing of a sterile neutrino with other active neutrinos can distort the standard oscillation probabilities. DUNE will be sensitive to this phenomenon through $v_{\mu}$ and $v_{e}$ spectra at both near and far detectors. It has been shown that the expected neutrino appearance and disappearance probabilities in the presence of sterile neutrinos are quite different from the standard ones [2]. An example result is displayed in the top-left panel of Figure 1, suggesting that DUNE will probe the relevant parameter space with sensitivities better than existing experiments.

The second example is CPT symmetry violation. In DUNE, CPT violation in the neutrino sector can be measured by analyzing neutrino and antineutrino oscillation parameters, separately. We have performed a sensitivity study for an exposure of $300 \mathrm{kt} \cdot \mathrm{MW} \cdot \mathrm{yr}$ and reported the sensitivities of DUNE to the difference in neutrino and antineutrino parameters, with $3 \sigma$ significance lines for reference. An example result [4] is shown in the top-right panel of Figure 1, from which we expect 

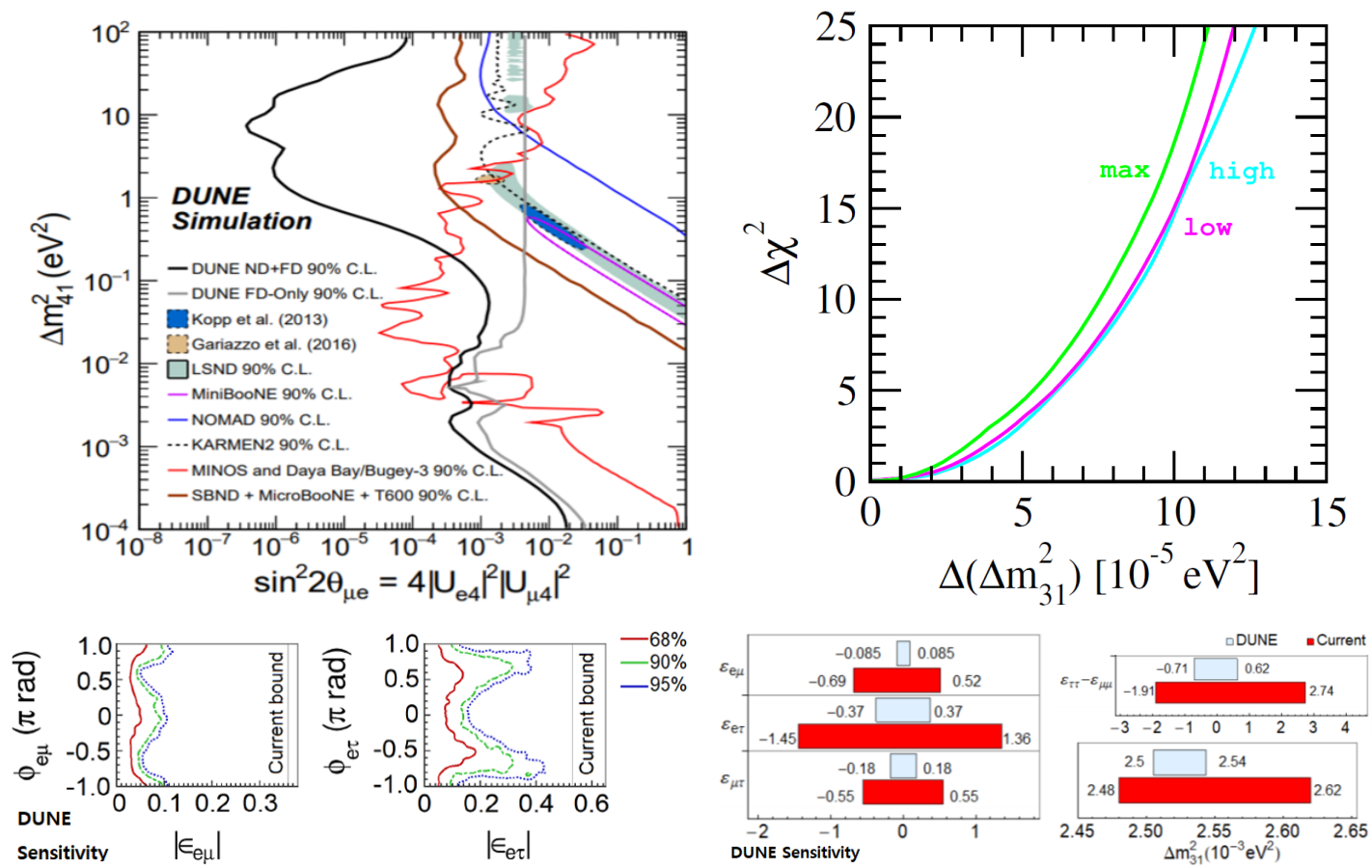

Figure 1: Top-left: DUNE sensitivities to $\sin ^{2} 2 \theta_{\mu e}$ from the appearance and disappearance samples at the near and far detectors, along with a comparison with previous existing experiments [2]. Top-right: The sensitivities of DUNE to the difference of a neutrino and antineutrino parameter $\Delta\left(\Delta m_{31}^{2}\right)$ for the atmospheric angle in the lower octant (magenta line), in the upper octant (cyan line), and for maximal mixing (green line) [4]. Bottom-left: Allowed regions (1.h.s. of the lines) for an exposure of $300 \mathrm{kt} \cdot \mathrm{MW} \cdot \mathrm{yr}$, along with a comparison with existing bounds [2]. Bottom-right: One-dimensional DUNE constraints (light blue) compared with current constraints (red) [2].

that DUNE can provide competitive bounds, in particular, put stronger bounds on $\Delta m_{31}^{2}$, improving the current limit by an order of magnitude.

A third new physics case is NSIs. We first focus on the NSIs in propagation via neutral current interactions. These NSIs can be described as new contributions to the Mikheyev-SmirnovWolfenstein effect. It has been shown that DUNE will improve current constraints especially on $\epsilon_{e \tau}$ and $\epsilon_{e \mu}$ by a factor of 2 to 5 [5-7]. For example, the plots in the bottom-left and the bottomright of Figure 1 show two-dimensional and one-dimensional DUNE constraints, respectively, in comparison with the existing bounds. These results suggest that DUNE can provide improved constraints on various NSI parameters and neutrino oscillation parameters.

\section{BSM at the Near Detector}

DUNE will be sensitive to beam-produced BSM signals leaving signatures in the near detector. The first example is the search for neutrino tridents. The neutrino trident is a very rare process, and previous experiments (e.g., CHARM-II [8]) observed just a few tens of events. In terms of BSM physics, a departure from the SM prediction can be considered as evidence of new physics, and it is shown that DUNE will be sensitive to the existence of light vector mediators in the trident 

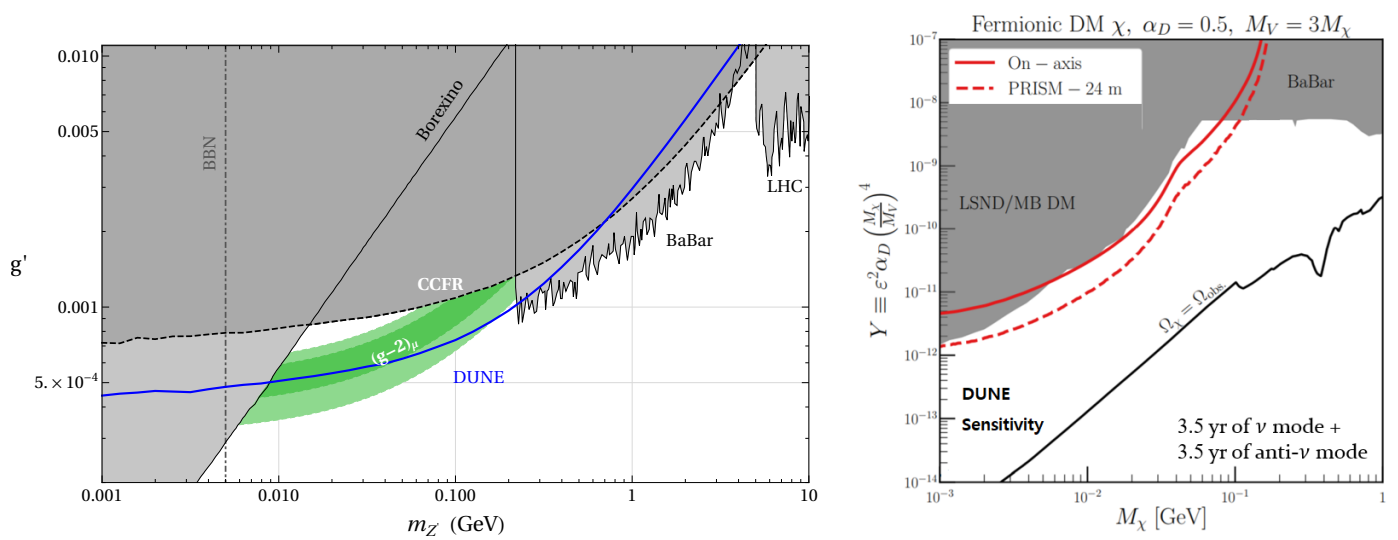

Figure 2: Left: Existing constraints and projected DUNE sensitivity in the $L_{\mu}-L_{\tau}$ parameter space [9]. Right: Expected DUNE On-axis (solid red) and PRISM (dashed red) sensitivity using $\chi e^{-} \rightarrow \chi e^{-}$scattering [2].

processes, for example, a $L_{\mu}-L_{\tau} Z^{\prime}$ which could explain the long-standing muon $g-2$ anomaly [9]. Sensitivity studies have been performed in the context of $Z^{\prime}$ models, for which an example result in the parameter plane of $Z^{\prime}$ mass $m_{Z^{\prime}}$ vs its gauge coupling $g^{\prime}$ is displayed in the left panel of Figure 2.

The second example is the search for a low-mass dark matter particle $\chi$. The main idea is that the highly intensified proton beam in DUNE can produce $\mathrm{MeV}$-range dark matter through meson decays into a dark photon $V$ which subsequently decays to dark matter in the target area, e.g., $\pi^{0} \rightarrow \gamma V, V \rightarrow \chi \chi$. Once produced, dark matter then scatters off an electron in the near detector, i.e., $\chi e^{-} \rightarrow \chi e^{-}$. Our sensitivity reaches in the plane of dark matter mass $M_{\chi}$ and an effective coupling $Y$ are shown in the right panel of Figure 2. Here two sensitivity curves are reported: one for on-axis mode and the other for off-axis mode (i.e., DUNE-PRISM). The sensitivity reach for the off-axis mode is better than that of the on-axis mode because the neutrino-induced background events can be suppressed in the off-axis mode [2]. Hence, DUNE-PRISM will be useful in the search for new physics.

\section{BSM at the Far Detector}

On top of the beam-induced signal, DUNE is expected to have decent sensitivities to cosmogenic new physics signals such as boosted dark matter. The first example is the search for inelastic boosted dark matter (iBDM) proposed in [10]. Models of iBDM hypothesize a two-component dark matter scenario where the dominant halo dark matter (say, $\chi_{0}$ ), which is usually the heavier component, pair-annihilates to a lighter dark matter pair (say, $\chi_{1}$ ) over the galaxy, resulting in boosting the lighter dark matter in the present universe. The boosted lighter dark matter then reaches the far detector and scatters off either an electron or a proton to an unstable dark-sector state (say, $\chi_{2}$ ) via an exchange of a dark photon (say, $X$ ) [10]. The $\chi_{2}$ then decays back to the $\chi_{1}$ together with additional visible particles, e.g., $\chi_{1} e^{-}(p) \rightarrow \chi_{2} e^{-}(p), \chi_{2} \rightarrow \chi_{1} X^{(*)}, X^{(*)} \rightarrow e^{-} e^{+}$. The DUNE far detector, which features a large fiducial mass and high resolution in energy, angle, and position, can be sensitive to these boosted dark matter signals with multiple particle tracks. In particular, 

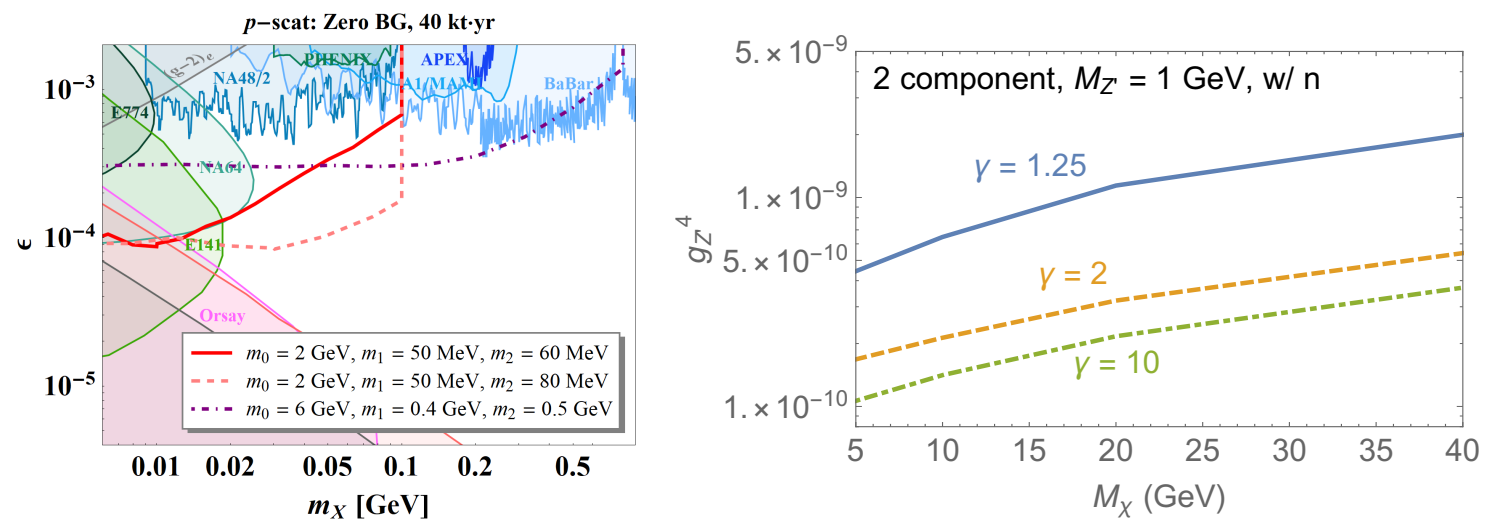

Figure 3: Left: Expected sensitivity reach of iBDM interacting with protons for three benchmark model points with an exposure of $40 \mathrm{kt} \cdot \mathrm{yr}$ [11]. Right: Expected $5 \sigma$ discovery reach of solar boosted dark matter with an exposure of $10 \mathrm{kt} \cdot y \mathrm{r}$ including neutrons in reconstruction [2].

a recent study [11] considered DUNE-like detectors with various detector effects parameterized appropriately. The left panel of Figure 3 shows an example result: the sensitivity reach in the dark photon parameter space. This suggests that DUNE has great potential in probing unexplored regions of this parameter space.

The second example is the search for solar boosted dark matter $[12,13]$. The main idea is similar to the previous one. However, the dominant dark matter component is captured in the Sun and annihilates to a pair of lighter dark matter components, or boosted dark matter is produced in the context of a semi-annihilation scenario. Unlike the galactic boosted dark matter, the source point is localized and close to the Earth. The DUNE far detector will be sensitive to these solar boosted dark matter signals, although they accompany only a target recoil; the angular resolution of the far detector allows us to infer the directionality of the signal events, and hence separate the signal from the backgrounds even if the signal of interest does not involve any additional signatures. The right panel of Figure 3 shows the DUNE sensitivity to the solar boosted dark matter interacting with nucleons via a $Z^{\prime}$ mediator. This suggests that DUNE can make a $5 \sigma$ discovery for an exposure of $10 \mathrm{kt} \cdot$ year [2].

\section{Conclusions}

BSM physics at neutrino facilities has been receiving increasing attention in recent years. In light of this situation, the DUNE experiment can provide excellent opportunities to test and study various BSM phenomenology. In particular, the high capability of the near and far DUNE detectors and the powerful beam source will allow for a rich experimental program of neutrino and nonneutrino BSM physics studies. We have briefly overviewed DUNE prospects of BSM related to the short-baseline and long-baseline neutrino oscillation phenomena, search for beam-induced new phenomena at the near detectors, and search for cosmic-origin new phenomena at the far detectors benefiting from their large mass and high resolution. 


\section{References}

[1] B. Abi et al. [DUNE], "Volume I. Introduction to DUNE," JINST 15, no.08, T08008 (2020) doi:10.1088/1748-0221/15/08/T08008 [arXiv:2002.02967 [physics.ins-det]].

[2] B. Abi et al. [DUNE], "Deep Underground Neutrino Experiment (DUNE), Far Detector Technical Design Report, Volume II DUNE Physics,” [arXiv:2002.03005 [hep-ex]].

[3] B. Abi et al. [DUNE], "Prospects for Beyond the Standard Model Physics Searches at the Deep Underground Neutrino Experiment," [arXiv:2008.12769 [hep-ex]].

[4] G. Barenboim, C. A. Ternes and M. Tórtola, "Neutrinos, DUNE and the world best bound on CPT invariance," Phys. Lett. B 780, 631-637 (2018) doi:10.1016/j.physletb.2018.03.060 [arXiv:1712.01714 [hep-ph]].

[5] T. Ohlsson, "Status of non-standard neutrino interactions," Rept. Prog. Phys. 76, 044201 (2013) doi:10.1088/0034-4885/76/4/044201 [arXiv:1209.2710 [hep-ph]].

[6] O. G. Miranda and H. Nunokawa, "Non standard neutrino interactions: current status and future prospects,” New J. Phys. 17, no.9, 095002 (2015) doi:10.1088/1367-2630/17/9/095002 [arXiv:1505.06254 [hep-ph]].

[7] Y. Farzan and M. Tortola, "Neutrino oscillations and Non-Standard Interactions," Front. in Phys. 6, 10 (2018) doi:10.3389/fphy.2018.00010 [arXiv:1710.09360 [hep-ph]].

[8] D. Geiregat et al. [CHARM-II], Phys. Lett. B 245, 271-275 (1990) doi:10.1016/03702693(90)90146-W

[9] W. Altmannshofer, S. Gori, J. Martín-Albo, A. Sousa and M. Wallbank, "Neutrino Tridents at DUNE," Phys. Rev. D 100, no.11, 115029 (2019) doi:10.1103/PhysRevD.100.115029 [arXiv:1902.06765 [hep-ph]].

[10] D. Kim, J. C. Park and S. Shin, "Dark Matter "Collider" from Inelastic Boosted Dark Matter," Phys. Rev. Lett. 119, no.16, 161801 (2017) doi:10.1103/PhysRevLett.119.161801 [arXiv:1612.06867 [hep-ph]].

[11] A. De Roeck, D. Kim, Z. G. Moghaddam, J. C. Park, S. Shin and L. H. Whitehead, "Probing Energetic Light Dark Matter with Multi-Particle Tracks Signatures at DUNE,” [arXiv:2005.08979 [hep-ph]].

[12] J. Berger, Y. Cui and Y. Zhao, "Detecting Boosted Dark Matter from the Sun with Large Volume Neutrino Detectors," JCAP 02, 005 (2015) doi:10.1088/1475-7516/2015/02/005 [arXiv:1410.2246 [hep-ph]].

[13] K. Kong, G. Mohlabeng and J. C. Park, "Boosted dark matter signals uplifted with self-interaction,” Phys. Lett. B 743, 256-266 (2015) doi:10.1016/j.physletb.2015.02.057 [arXiv:1411.6632 [hep-ph]]. 